\title{
Identification of Novel Metabolites of Vildagliptin in Rats: Thiazoline- Containing Thiol Adducts Formed via Cysteine or Glutathione Conjugation ${ }^{\mathbb{S}}$
}

\author{
Katsuhiko Mizuno, Kenji Takeuchi, Ken Umehara, and Miki Nakajima
}

\begin{abstract}
Department of Drug Metabolism and Pharmacokinetics, Nonclinical Research Center, Tokushima Research Institute, Otsuka Pharmaceutical Co., Ltd., Kawauchi-cho, Tokushima, Japan (K.M., K.T.); Nonclinical Research Center, Tokushima Research Institute, Otsuka Pharmaceutical Co., Ltd., Kawauchi-cho, Tokushima, Japan (K.U.); Drug Metabolism and Toxicology, Faculty of Pharmaceutical Sciences, Kanazawa University, Kakuma-machi, Kanazawa Japan (K.M., M.N.); and WPI Nano Life Science Institute
\end{abstract} (WPI-NanoLSI), Kanazawa University, Kakuma-machi, Kanazawa, Japan (M.N.)

Received January 24, 2019; accepted May 14, 2019

\section{ABSTRACT}

Vildagliptin (VG), a dipeptidyl peptidase-4 inhibitor, is used for treating type 2 diabetes. On rare occasions, VG causes liver injury as an adverse reaction. One case report suggested the involvement of immune responses in the hepatotoxicity, but the underlying mechanisms are unknown. We recently reported that VG binds covalently in vitro to L-cysteine to produce a thiazoline acid metabolite, M407, implying that the covalent binding may trigger the immune-mediated hepatotoxicity. There was no evidence, however, that such a thiazoline acid metabolite was formed in vivo. In the present study, we administered a single oral dose of VG to male Sprague-Dawley rats, and detected M407 in plasma. The sum of urinary and fecal excretions of M407 reached approximately
$2 \%$ of the dose 48 hours postdosing. Using bile duct-cannulated rats, we demonstrated that M407 was secreted into bile as a glucuronide, designated as M583. Another newly identified thiazoline metabolite of VG, the cysteinylglycine conjugate M464, was detected in urine, feces, and bile. The formation of M464 was confirmed by in vitro incubation of VG with glutathione even in the absence of metabolic enzymes. A glutathione adduct against the nitrile moiety M611 was also detected in vitro but not in vivo. In summary, we found three new thiazoline-containing thiol adduct metabolites in VG-administered rats. Nonenzymatic covalent binding of VG would likely occur in humans, and it may be relevant to predicting adverse reactions.

\section{Introduction}

Vildagliptin (VG) is a potent, selective, and orally administratable dipeptidyl peptidase-4 inhibitor (Villhauer et al., 2003), and is used to treat type 2 diabetes mellitus worldwide (Deacon and Lebovitz, 2016). VG is generally considered a safe medication because it does not increase the risk of any adverse events of interest, such as infections and hepatotoxicity, or cardiovascular events (Ligueros-Saylan et al., 2010; Mathieu et al., 2017). Rare cases of hepatic dysfunction (including hepatitis) have, however, been reported (http://www.emea.europa.eu/ docs/en_GB/document_library/EPAR_-_Product_Information/human/ 000771/WC500020327.pdf). In Japan, a case of liver injury with elevated serum transaminase levels was observed after an 8-month VG treatment of $50 \mathrm{mg} /$ day (Kurita et al., 2014). In this case, a druginduced lymphocyte stimulation test (DLST) was positive, indicating that immune responses were associated with the liver injury. Another DLST-positive case, in which no symptoms of hepatotoxicity were observed, presented with fever and acute inflammation resulting from hypercytokinemia after a third dose of VG (100 mg/day) (Anno et al.,

No potential conflicts of interest relevant to this article are reported. https://doi.org/10.1124/dmd.119.086546.

S This article has supplemental material available at dmd.aspetjournals.org.
2019). In preclinical studies of VG, no hepatic abnormalities were observed (http://www.emea.europa.eu/docs/en_GB/document_library/ EPAR_-_Product_Information/human/000771/WC500020327.pdf) (Busch et al., 2013). The molecular mechanisms of VG-induced liver injury or hypersensitivity in humans are unknown.

Immune-mediated drug-induced liver injury (DILI) appears to be initiated by irreversible covalent binding of a drug or its metabolites. Reactive metabolites formed by drug-metabolizing enzymes such as cytochrome P450 bind directly to cellular macromolecules. Some drugs themselves, such as penicillin and aspirin, covalently bind to proteins in a nonspecific manner. Drug-protein adducts, including haptens, acquire immunogenicity as neoantigens and trigger immune responses. Nucleophilic sites of proteins, such as the thiol residues of cysteine, are the target of electrophiles; these electrophiles can be trapped by thiol residues of glutathione (GSH) or L-cysteine. Drug conjugates with GSH or cysteine, as well as their mercapturic acid metabolites, are considered markers of covalent binding (Stepan et al., 2011; Gómez-Lechón et al., 2016).

In both healthy volunteers and patients with type 2 diabetes mellitus, VG is absorbed rapidly and well after oral administration (He, 2012). VG is extensively metabolized by hydrolysis of a nitrile moiety to form the carboxylic acid metabolite M20.7 in humans (He et al., 2009a), and human dipeptidyl peptidase- 4 greatly contributes to the hydrolysis of

ABBREVIATIONS: CDNB, 1-chloro-2,4-dinitrobenzene; DILI, drug-induced liver injury; DLST, drug-induced lymphocyte stimulation test; EA, ethacrynic acid; GSH, glutathione; GST, glutathione S-transferase; HLC, human liver cytosol; IS, internal standard; LC-MS, liquid chromatographymass spectrometry; LC-MS/MS, liquid chromatography-tandem mass spectrometry; $\mathrm{m} / \mathrm{z}$, mass/charge ratio; VG, vildagliptin. 
VG (Asakura et al., 2015). In addition, minor metabolites from the hydrolysis of an amide bond (M15.3), glucuronidation (M20.2), or oxidation on the pyrrolidine moiety of VG (M20.9 and M21.6) have been identified (He et al., 2009a). These metabolites have also been detected in animals (He et al., 2009b; Asakura et al., 2017). GSHconjugated metabolites and their derivatives, however, have not been found in either humans or animals. Dahal et al. (2013) reported that radiolabeled VG binds to macromolecules in human hepatocytes in vitro, suggesting that VG can form covalent bonds. We recently found that VG binds covalently to the thiol residue of $\mathrm{L}$-cysteine rather than to GSH (Mizuno et al., 2018). Structural analysis of the compound revealed that the nitrile moiety of $\mathrm{VG}$ is irreversibly converted to a thiazoline acid to form the VG-cysteine adduct M407 (Fig. 1). Analogous covalent binding to protein thiols may initiate immunemediated reactions, leading to hepatotoxicity. In this study, to determine whether this thiol adduct can be formed in vivo, we administered VG orally to rats, and measured the formation of metabolites.

\section{Materials and Methods}

Chemicals and Reagents. VG and its carboxylic acid metabolite (M20.7) were purchased from LKT Laboratories (St. Paul, MN) and Santa Cruz Biotechnology (Dallas, TX), respectively. M407 was previously synthesized (Mizuno et al., 2018). GSH was purchased from Nacalai Tesque (Kyoto, Japan). 1-Chloro-2,4-dinitrobenzene (CDNB) and ethacrynic acid (EA) were purchased from Tokyo Chemical Industry (Tokyo, Japan). Human liver cytosol (HLC; Ultrapool Human Cytosol 150) and $\beta$-glucuronidase solution (from Escherichia coli K12) were obtained from Corning (Corning, NY) and Roche Diagnostics (Risch-Rotkreuz, Switzerland), respectively. Other chemicals and reagents were of analytical grade or the highest grade commercially available.

Animals. All animal studies were conducted with the approval of the Animal Ethical Committee of Otsuka Pharmaceutical Co., Ltd. Male Sprague-Dawley rats and bile duct-cannulated Sprague-Dawley rats were purchased from Charles River Laboratories Japan (Kanagawa, Japan). All animals had free access to food and water and were not fasted before dosing.

Pharmacokinetic Studies. Rats (260-290 g, 7 weeks old, $n=6$ ) were orally administered VG at a dose of $30 \mathrm{mg} / \mathrm{kg}(5 \mathrm{ml} / \mathrm{kg})$, which was dissolved in $0.5 \%$ carboxymethyl cellulose/ $0.2 \%$ Tween 80 , following the method of Burkey et al. (2005), without anesthesia. Blood samples were sequentially collected from three individual rats $1,2,4,8,24$, and 48 hours postdosing and centrifuged at $1800 \mathrm{~g}$ at $4^{\circ} \mathrm{C}$ for 10 minutes to obtain plasma. The remaining three rats were placed in individual metabolic cages, and urine and feces were collected in 24-hour intervals up to 48 hours postdosing. Bile duct-cannulated rats (290-330 g, 8 weeks old, $n=3$ ) were also orally administered $30 \mathrm{mg} / \mathrm{kg}$ VG. In addition to urine and feces, bile was collected via cannula $0-4,4-8,8-24$, and $24-48$ hours postdosing. The fecal samples were homogenized in a ninefold weight of water by shaking. The plasma, urinary, fecal homogenate, and biliary samples were stored at or below $15^{\circ} \mathrm{C}$ until analysis.

Quantification of VG and Metabolites by Liquid Chromatography-Tandem Mass Spectrometry. The plasma, urinary, fecal homogenate, and biliary samples were mixed with 4-, 10-, 10-, and 50-fold volume of ice-cold acetonitrile containing $1 \mathrm{ng} / \mathrm{ml}$ spiperone as an internal standard (IS), respectively. After centrifugation at $6130 \mathrm{~g}$ at $4^{\circ} \mathrm{C}$ for 10 minutes, the supernatant was subjected to liquid chromatography-tandem mass spectrometry (LC-MS/MS) analysis. The LC-MS/MS system consisted of a Prominence UFLC System (Shimadzu, Kyoto, Japan) and an API4000 Triple-Quadrupole Mass Spectrometer equipped with an electrospray ionization source (AB SCIEX, Framingham, MA) and a switching valve (Valco Instruments, Houston, TX). Analyte separation was achieved on an XBridge $\mathrm{C} 18$ column $(3.5 \mu \mathrm{m} ; 2.1 \times 50 \mathrm{~mm}$ i.d.; Waters, Milford, MA). The two mobile phases consisted of $0.5 \mathrm{mM}$ ammonium hydrogen carbonate aqueous solution (A) and methanol (B). The flow rate was $0.55 \mathrm{ml} / \mathrm{min}$. Linear-gradient elution was performed as follows: $5 \% \mathrm{~B}$ at $0-0.1$ minute, $5 \%-90 \% \mathrm{~B}$ at 0.1-1.0 minute, and $90 \% \mathrm{~B}$ at 1.0-2.0 minutes. The autosampler tray was maintained at $4{ }^{\circ} \mathrm{C}$. The mass spectrometer was operated in electrospray-positive ion mode using multiple reaction monitoring transitions at mass/charge ratio $(\mathrm{m} / \mathrm{z})$ 304.2/154 for VG, 323.2/173 for M20.7, 408.2/258 for M407, and 396.0/165 for

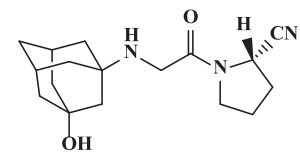

Vildagliptin (VG)

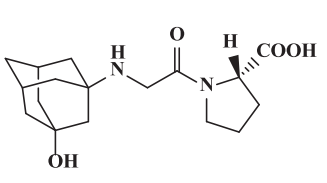

M20.7

(major metabolite of VG)

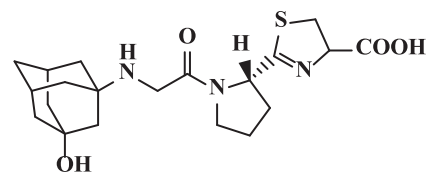

M407

(cysteine conjugate of $\mathrm{VG}$ )
Fig. 1. Chemical structures of VG, its major metabolite M20.7, and the cysteineconjugated metabolite M407.

spiperone. Quantification of each analyte was achieved by comparing the analyte/IS peak area ratios to those of calibration curves ranging from 0.003 to $0.6 \mu \mathrm{M}$ for plasma, 0.1 to $20 \mu \mathrm{M}$ for urine, $1-200 \mathrm{nmol} / \mathrm{g}$ feces for fecal homogenate, and 0.05 to $10 \mu \mathrm{M}$ for bile.

In addition, deconjugation of the biliary samples via $\beta$-glucuronidase was conducted to estimate the amount of the conjugated metabolites. The biliary sample was mixed with a fivefold volume of $0.1 \mathrm{~mol} / \mathrm{l}$ sodium phosphate buffer $(\mathrm{pH}$ 6.0) and a fivefold volume of the $\beta$-glucuronidase solution (at least $140 \mathrm{U} / \mathrm{ml}$ ). After incubation at $37^{\circ} \mathrm{C}$ for 24 hours, an aliquot of the deconjugated sample was collected and mixed with a 4.5 -fold volume of ice-cold acetonitrile containing the IS, followed by the deproteination and LC-MS/MS analysis as described above.

Data Analysis for Plasma Pharmacokinetics and Excretions. The pharmacokinetics parameters $C_{\max }$, time to reach $C_{\max }$, area under the plasma concentration time curve from time zero to infinity, and terminal-phase elimination half-life of each analyte were calculated from plasma concentrationtime profiles using a noncompartmental model in Phoenix WinNonlin version 6.4 (Pharsight Corp., St. Louis, MO). Analytes of urinary, fecal, and biliary excretions (in nanomoles) were calculated by multiplying the concentration (micromoles or nanomoles per gram) by the volume (milliliters or grams). Excretion, as a percentage of the dose, up to 48 hours postdosing, was calculated from the ratio of the sum of the excretion amount at each period to the dose.

Identification of Metabolites by Liquid Chromatography-Mass Spectrometry. The plasma, urinary, fecal homogenate, and biliary samples collected from three individual rats were pooled at each time point and mixed with a twofold volume of ice-cold acetonitrile-methanol (1:1, v/v). After centrifugation at $20,400 \mathrm{~g}$ at $4^{\circ} \mathrm{C}$ for 10 minutes, the supernatant was subjected to liquid chromatography-mass spectrometry (LC-MS) analysis using an Orbitrap Fusion MS System coupled with an UltiMate 3000 HPLC System (Thermo Fisher Scientific, Waltham, MA). Analytes were separated using an XBridge C18 Column $(5 \mu \mathrm{m} ; 4.6 \times 100 \mathrm{~mm}$ i.d.; Waters $)$ maintained at $25^{\circ} \mathrm{C}$ and eluted with $1.0 \mathrm{ml} / \mathrm{min}$ mobile phases (A, $20 \mathrm{mM}$ ammonium acetate aqueous solution; $\mathrm{B}$, acetonitrile) with a linear gradient of $5 \% \mathrm{~B}$ at 0 minute, $10 \% \mathrm{~B}$ at 2 minutes, and $90 \%$ B at 8 minutes. The Orbitrap Fusion System was operated to obtain full-scan mass spectra and product-ion spectra over the range of $\mathrm{m} / \mathrm{z} 100$ to 1000 in electrospray-positive and electrospray-negative ionization modes. First, referring to previous reports (He et al., 2009b; Mizuno et al., 2018), exact ion chromatograms of $[\mathrm{M}+\mathrm{H}]^{+}$and $[\mathrm{M}-\mathrm{H}]^{-}$ions of $\mathrm{VG}$ and the identified metabolites (M15.3, M20.7, M20.2, and two types of mono-hydroxyl VG, M18.6, and M407) were obtained. When the specified mass peaks were detected on the exact ion chromatograms, the product-ion spectra were analyzed to confirm their chemical structures. Next, to locate unidentified thiol adduct metabolites, specified peaks in VG-administered samples were searched on the full-scan mass spectra by comparing with mass spectra from control samples obtained from untreated rats. For each peak, the structure was estimated from the accurate masses of precursor- and product-ion spectra to assess whether the product was a thiol adduct metabolite of VG.

In Vitro Approaches to Evaluate GSH Adducts of VG by LC-MS. VG $(100 \mu \mathrm{M})$ was incubated with or without GSH $(100 \mathrm{mM})$ in a potassium phosphate 
A

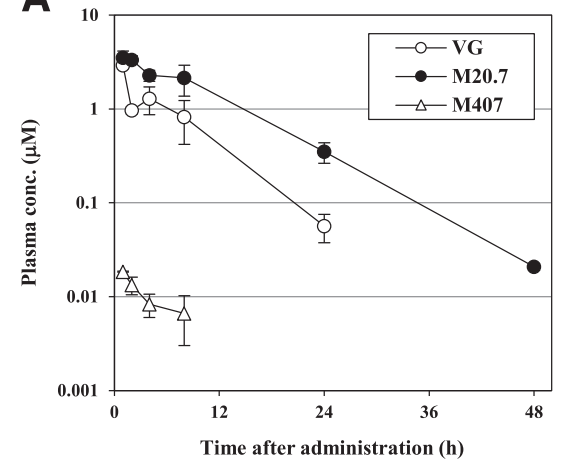

B

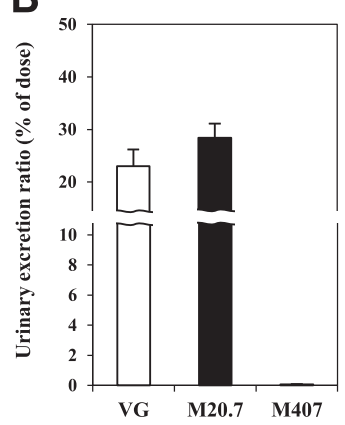

C

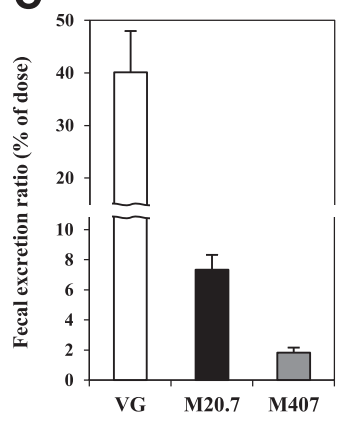

Fig. 2. Plasma concentration-time profile and urinary and fecal excretions after a single oral dose of $30 \mathrm{mg} / \mathrm{kg}$ VG to male Sprague-Dawley rats. (A) Blood was drawn at 1, 2, 4, 8, 24, and 48 hours postdosing to measure plasma concentrations of VG and its metabolites M20.7 and M407. Urinary (B) and fecal (C) excretions were measured up to 48 hours postdosing. Data are shown as the mean \pm S.D. of three rats. buffer $(100 \mathrm{mM}, \mathrm{pH} 7.4)$ at $37^{\circ} \mathrm{C}$ for 24 hours. Aliquots of the reaction mixture were analyzed using an Orbitrap Fusion MS System, as described above. When the product peaks were detected at $207 \mathrm{~nm}$, their chemical structures were estimated from the precursor- and product-ion spectra.

Effects of Human GSH $\boldsymbol{S}$-Transferase on GSH Adduct Production from VG. Incubation mixtures consisted of $1 \mathrm{mg} / \mathrm{ml} \mathrm{HLC}, 100 \mu \mathrm{M} \mathrm{VG}, 5 \mathrm{mM} \mathrm{GSH}$, and $100 \mu \mathrm{M}$ EA in a total volume of $300 \mu \mathrm{l}$ of Tris- $\mathrm{HCl}(100 \mathrm{mM}, \mathrm{pH} 7.5)$. The final fraction of ethanol in the mixture was $0.3 \%$. After preincubation for 2 minutes, the reaction was started by adding GSH in a shaking water bath at $25^{\circ} \mathrm{C}$. After incubation for 30 minutes, $300 \mu \mathrm{l}$ of ice-cold acetonitrile containing $1 \mathrm{ng} / \mathrm{ml}$ spiperone as an IS was added to terminate the reaction. After centrifugation at $6130 \mathrm{~g}$ at $4{ }^{\circ} \mathrm{C}$ for 10 minutes, the supernatant was subjected to LC-MS/MS analysis as described previously. The multiple reaction monitoring transitions of M464 and M611 were $m / z$ 465.2/137 and 612.3/179, respectively. The relative concentration of each analyte was calculated by comparing the analyte/IS peak area ratios.

To evaluate whether GSH $S$-transferase (GST) and EA work adequately in the present assay, GSH adduct formation from CDNB induced by $\mathrm{HLC}$ and the effect of EA on the reaction were determined. After preincubation for 2 minutes, $200 \mu \mathrm{M}$ CDNB was incubated with $0.01 \mathrm{mg} / \mathrm{ml} \mathrm{HLC}, 5 \mathrm{mM} \mathrm{GSH}$, and $100 \mu \mathrm{M}$ EA in Tris- $\mathrm{HCl}(100 \mathrm{mM}, \mathrm{pH} 7.5,0.3 \%$ ethanol $)$ at $25^{\circ} \mathrm{C}$. After incubation for 5 minutes, the UV absorbance at $340 \mathrm{~nm}$ was measured using a plate reader (SpectraMax M5; Molecular Devices Japan, Tokyo, Japan) to calculate the concentration of the CDNB-GSH adduct (extinction coefficient, $9.6 \mathrm{mM} / \mathrm{cm}$ (Habig et al., 1974).

Statistical Analysis. Data are expressed as the mean \pm S.D. Statistical analyses were carried out using an unpaired $t$ test or one-way analysis of variance followed by Tukey's test, and $P<0.05$ was considered significant.

\section{Results}

Plasma Pharmacokinetics and Excretions of VG and Its Metabolites in Rats. VG was orally administered at a dose of $30 \mathrm{mg} / \mathrm{kg}$ to fed male rats, and the plasma concentration-time profiles and excretions into urine and feces of VG and its metabolites M20.7 and M407 were calculated. M20.7, a carboxylic acid metabolite of VG, is its major metabolite in humans and animals (He et al., 2009a,b; Asakura et al., 2015). M407 is a thiazoline acid metabolite formed via cysteine conjugation, which we identified previously (Mizuno et al., 2018). After administration of $\mathrm{VG}, \mathrm{M} 20.7$ was detected in plasma at higher concentrations than VG (Fig. 2A). The pharmacokinetic parameters of M20.7 and VG (Table 1) were consistent with those reported in a previous study, in which $100 \mathrm{mg} / \mathrm{kg}\left[{ }^{14} \mathrm{C}\right] \mathrm{VG}$ was orally administered to fed male Wistar Han rats (He et al., 2009b). M407 was detected in the plasma at approximately $1 \%$ of VG, indicating that M407 is formed from VG not only in vitro but also in vivo (Fig. 2A; Table 1). In addition to VG and M20.7, M407 was also excreted into urine and feces (Fig. 2, B and C). M407 was preferentially excreted into feces, and the total excretion reached $2 \%$ of the dose. The sum of urinary excretion of VG and the two metabolites reached $50 \%$ of the dose, suggesting that the oral bioavailability of VG was $>50 \%$. Total excretion of VG and the two metabolites until 48 hours postdosing reached approximately $100 \%$ of the dose.

To confirm whether the M407 detected in feces had been formed in the body, the biliary samples collected from bile duct-cannulated rats that received $\mathrm{VG}$ were subjected to analysis. The urinary excretions of VG, M20.7, and M407 in the bile duct-cannulated rats (Fig. 3A) were comparable with those of normal rats (Fig. 2B). Excretions of VG, M20.7, and M407 into feces (Fig. 3B) were lower than those in normal rats (Fig. 2C). In addition to VG and M20.7, M407 was detected in bile (Fig. 3C). Accordingly, we confirmed that M407 is actually formed in vivo.

VG Metabolites in Rat Plasma, Urine, Feces, and Bile. To identify other thiol adduct metabolites, we analyzed the plasma, urinary, fecal, and biliary samples from normal or bile duct-cannulated rats by LC-MS. Since no peak was observed by monitoring at $207 \mathrm{~nm}$, the maximum absorption wavelength of VG (data not shown), we searched for metabolites by scanning over the range of $\mathrm{m} / \mathrm{z} 100$ to 1000 in the positive and negative ion modes. M15.3, M20.7, M20.2, M18.6, and two types of mono-hydroxyl VG (either M14.9 or M16.7), which are all known VG metabolites in rats (He et al., 2009b) were detected in rat plasma, urine, feces, and bile, with the exception of M15.3 in plasma and M20.2 in feces (Supplementary Fig. 1; Table 2). The structures of these metabolites are shown in Fig. 4. In addition, two peaks were detected at 2.5 and 4.0 minutes in bile, and named M583 and M464, respectively. The latter peak was also detected in urine and feces. The estimated molecular weight of M583 was $176\left(\mathrm{C}_{6} \mathrm{H}_{8} \mathrm{O}_{6}\right)$ higher than that of M407, indicating that M583 was a glucuronide of M407. As shown in Fig. 5A, fragmentation of the pseudomolecular ion $[\mathrm{M}+\mathrm{H}]^{+}$of $\mathrm{M} 583$ yielded $\mathrm{m} / \mathrm{z}$ $258,241,201,151$, and 70 , and that of $[\mathrm{M}-\mathrm{H}]^{-}$yielded ions at $\mathrm{m} / \mathrm{z}$ 362 and 155, characteristic of M407 (Mizuno et al., 2018). The ions at $\mathrm{m} / \mathrm{z} 538$ and 354 formed by $[\mathrm{M}-\mathrm{H}]^{-}$corresponded to the loss of a carboxylic acid from M583 and a glucuronide of hydroxyadamantane moiety, respectively, indicating that M583 was an ether-linked $\mathrm{O}$-glucuronide but not an acyl glucuronide, unlike M20.2 (Fig. 5B). The estimated molecular weight of M464 was $57\left(\mathrm{C}_{2} \mathrm{H}_{3} \mathrm{NO}\right)$ higher than that of $\mathrm{M} 407$, and the difference corresponded to a dehydrated glycine. As shown in Fig. 5C, fragmentation of the $[\mathrm{M}+\mathrm{H}]^{+}$of M464 resulted in signals at $m / z 315,298$, and 258 , which correspond to the product ions of M407 ( $\mathrm{m} / \mathrm{z}, 258,241$, and 201) with a difference of $\mathrm{m} / \mathrm{z} 57$ (Mizuno et al., 2018). The ion $[\mathrm{M}-\mathrm{H}]^{-}$of M464 produced $\mathrm{m} / \mathrm{z}, 74$ corresponding to a glycine, indicating that M464 has a glycine moiety in its structure. Accordingly, M464 was estimated to be a cysteinylglycine conjugate of VG, which has a thiazoline moiety, as does M407 (Fig. 5D).

Quantification of M583 Biliary Excretions. M583, the glucuronide of M407, was detected in bile but not in feces (Table 2); therefore, we hypothesized that M583 excreted into bile would be converted to M407 
TABLE 1

Plasma pharmacokinetics parameters after a single oral administration of $30 \mathrm{mg} / \mathrm{kg}$ VG to male Sprague-Dawley rats Data are shown as the mean \pm S.D. of three rats.

\begin{tabular}{lcccc}
\hline Compound & \multicolumn{1}{c}{$C_{\max }(\mu \mathrm{M})$} & \multicolumn{1}{c}{$\mathrm{t}_{\max }(\mathrm{h})$} & $\mathrm{AUC}_{\text {inf }}(\mu \mathrm{M} \mathrm{h})$ & $t_{1 / 2, \mathrm{z}}(\mathrm{h})$ \\
\hline VG & $2.919 \pm 0.144$ & $1.00 \pm 0$ & $17.28 \pm 4.65$ & $4.535 \pm 1.099$ \\
M20.7 & $3.710 \pm 0.534$ & $1.33 \pm 0.58$ & $44.19 \pm 7.54$ & $6.040 \pm 0.490$ \\
M407 & $0.001847 \pm 0.00017$ & $1.00 \pm 0$ & $0.1514 \pm 0.0946$ & $6.895 \pm 3.817$ \\
\hline
\end{tabular}

$\mathrm{AUC}_{\mathrm{inf}}$, area under the plasma concentration-time curve from time zero to infinity; $t_{1 / 2, z}$, terminal-phase elimination half-life; $\mathrm{t}_{\mathrm{max}}$, time to reach $C_{\max }$.

by intestinal $\beta$-glucuronidase. Accordingly, rat bile was treated with $\beta$-glucuronidase, and the sample was subjected to metabolite analysis. M20.2, the glucuronide of VG, was also evaluated because it was detected in bile but not in feces (Table 2). After incubation with $\beta$-glucuronidase, M407 and VG contents increased significantly (Table 3). Based on the difference in M407 and VG contents after treatment of bile with $\beta$-glucuronidase and no treatment with $\beta$-glucuronidase, M583 and M20.2 excretion was estimated to be $0.1 \%$ and $33 \%$ of the dose, respectively. The results suggested that M583 was excreted into bile and then deconjugated by $\beta$-glucuronidase to M407 in the intestine.

Formation of M464 and M611 by In Vitro Incubation of VG with GSH. Since M464 was estimated to be a cysteinylglycine conjugate containing a thiazoline moiety (Fig. 5D), we assumed that M464 would be formed by covalent binding of GSH prior to deammoniation and hydrolysis of the glutamine-cysteine bond, similar to the mechanism of M407 formation by L-cysteine (Mizuno et al., 2018). To test the hypothesis, VG was incubated with $\mathrm{GSH}$ at $37^{\circ} \mathrm{C}$ in the absence of human liver enzymes, which are not essential for the formation of M407 (Mizuno et al., 2018). Since our previous study indicated that VG is stable during incubation with GSH (half-life, $>2$ hours), we set the incubation time at 24 hours in this study. Two peaks were observed at 3.68 and 4.18 minutes by UV light detection at $207 \mathrm{~nm}$ (Fig. 6A). The precursor-ion and product-ion spectra of the peak at 4.18 minutes were consistent with those of M464 detected in rat urine, feces, and bile (data not shown). We therefore confirmed that M464 is formed by covalent binding of GSH to VG.

Another peak at 3.68 minutes was also estimated in the same manner. This peak had a $[\mathrm{M}+\mathrm{H}]^{+}$of $m / z 612.2703\left(\left[\mathrm{C}_{27} \mathrm{H}_{42} \mathrm{~N}_{5} \mathrm{O}_{9} \mathrm{~S}\right]^{+} ; \Delta, 0.5\right.$ $\mathrm{mDa})$ and a $[\mathrm{M}-\mathrm{H}]^{-}$of $\mathrm{m} / z 610.2549\left(\left[\mathrm{C}_{27} \mathrm{H}_{40} \mathrm{~N}_{5} \mathrm{O}_{9} \mathrm{~S}\right]^{-} ; \Delta,-0.3\right.$ $\mathrm{mDa}$ ) in positive and negative ionization modes, respectively. We therefore named this compound M611. As shown in Fig. 6B, the peak of $[\mathrm{M}+\mathrm{H}]^{+} \mathrm{m} / \mathrm{z}, 612$ yielded product ions at $\mathrm{m} / \mathrm{z} 179$ and 162 , and the peak of $[\mathrm{M}-\mathrm{H}]^{-} \mathrm{m} / \mathrm{z} 610$ yielded product ions at $\mathrm{m} / \mathrm{z}, 306,272,254,143$, and 128 , which are characteristic of GSH. Considering the product ions at $\mathrm{m} / \mathrm{z} 259$ and 321 from $[\mathrm{M}+\mathrm{H}]^{+}$and $\mathrm{m} / \mathrm{z} 321$ and 337 from $[\mathrm{M}-\mathrm{H}]^{-}$, M611 was estimated to be a GSH conjugate without the thiazoline ring formation shown in Fig. 6C, although this metabolite and its subsequent metabolites, such as cysteine adducts or mercapturic acid metabolites, were not detected in vivo (data not shown). Therefore, M611 formation may be limited to in vitro conditions, or M611 may be extensively degraded in vivo.

Effects of Human GST on GSH Adduct Production from VG. Because GST contributes to the production of GSH-conjugated metabolites of drugs (Hayes et al., 2005), the effects of human cytosolic GST on M464 and M611 production were evaluated. As shown in Fig. 7A, M464 formation from VG was not increased by coincubation with HLC and GSH. In addition, the GST inhibitor EA did not affect the formation. M611 was not detected because of low production (data not shown). Levels of the GSH adduct production of CDNB, a probe substrate of GST, significantly increased in the presence of HLC (Fig. 7B) with sufficient GST activity $(458 \pm 69 \mathrm{nmol} / \mathrm{min}$ per milligram cytosolic protein). EA greatly inhibited GST activity $(34.1 \pm 4.2 \mathrm{nmol} /$ min per milligram), indicating that cytosolic GST and EA worked adequately in the experiment. The results suggested that human GST hardly contributes to the GSH conjugation of VG.

\section{Discussion}

The occurrence of DILI is a common issue in drug development and the most frequent reason for postmarketing warnings and withdrawals. In many cases, idiosyncratic DILI cannot be detected in early-stage clinical trials because adverse events occur in a small minority of susceptible patients. Rare hepatotoxic events lead to severe hepatic failures or mortalities in later clinical trials or postmarketing (Lee, 2003; Kaplowitz, 2005). Idiosyncratic reactions are classified as allergic or nonallergic; allergic reactions involve the adaptive immune system, and are characterized as fever, rash, production of autoantibodies, and the rapid recurrence of hepatotoxicity on drug rechallenge. The DLST is used to identify the involvement of this mechanism. Immune-mediated idiosyncratic DILI may be attributed to multiple mechanisms, such as covalent binding of chemicals to macromolecules and the release of danger signals from innate immune cells or injured hepatocytes. Covalent binding of a drug or its reactive metabolite to proteins forms a hapten, which induces immune responses. The risk of covalent binding is a major concern in drug development in the pharmaceutical industries
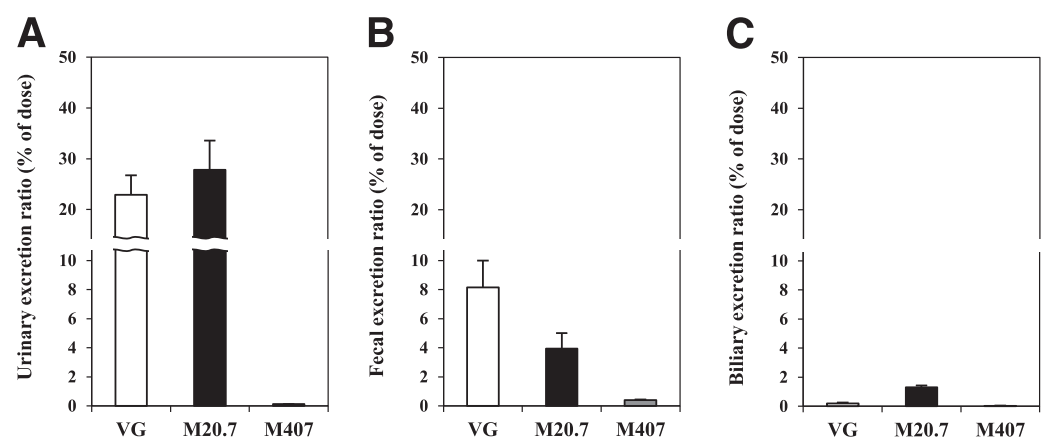

Fig. 3. Urinary, fecal, and biliary excretions of VG and its metabolites M20.7 and M407 after a single oral administration of $30 \mathrm{mg} / \mathrm{kg}$ VG to bile duct-cannulated rats. Urine (A), feces (B), and bile (C) were collected up to 48 hours postdosing to measure the excretion of VG, M20.7, and M407. Data are shown as the mean \pm S.D. of three rats. 
TABLE 2

Metabolites in rat plasma, urine, feces, and bile after a single oral dose of VG

\begin{tabular}{|c|c|c|c|c|c|c|}
\hline Peak & $\begin{array}{l}\text { Retention } \\
\text { Time (min) }\end{array}$ & Normal Rats & Bile Duct-Cannulated Rats & $\begin{array}{l}\text { Polarity, Accurate Masses and } \\
\Delta \text { of }[\mathrm{M}+\mathrm{H}]^{+} \text {and }[\mathrm{M}-\mathrm{H}]^{-}\end{array}$ & Estimated Formula & Identified or Estimated Structure \\
\hline & & & & $\mathrm{Da}(\mathrm{mDa})$ & & \\
\hline M15.3 & $2.06-2.12$ & $\mathrm{U}, \mathrm{F}$ & $\mathrm{U}, \mathrm{F}, \mathrm{B}$ & $\begin{array}{l}\text { Pos: } 226.1432-9(-0.6 \text { to } 0.1) \\
\quad \text { Neg: not detectable }\end{array}$ & $\mathrm{C}_{12} \mathrm{H}_{19} \mathrm{NO}_{3}$ & Carboxylic acid metabolite of VG \\
\hline M583 & $\begin{array}{l}2.0-2.9 \\
\text { (broad) }\end{array}$ & & B & $\begin{array}{l}\text { Pos: } 584.2267(-0.5) \\
\quad \text { Neg: } 582.2117(-1.0)\end{array}$ & $\mathrm{C}_{26} \mathrm{H}_{37} \mathrm{~N}_{3} \mathrm{O}_{10} \mathrm{~S}$ & $O$-Glucuronide of M407 \\
\hline M20.7 & $\begin{array}{l}2.0-3.0 \\
\quad \text { (broad) }\end{array}$ & $\mathrm{P}, \mathrm{U}, \mathrm{F}$ & $\mathrm{U}, \mathrm{F}, \mathrm{B}$ & $\begin{array}{l}\text { Pos: } 323.1958-66(-0.7 \text { to } 0.1) \\
\quad \text { Neg: } 321.1805-20(-1.5 \text { to } 0)\end{array}$ & $\mathrm{C}_{17} \mathrm{H}_{26} \mathrm{~N}_{2} \mathrm{O}_{4}$ & Carboxylic acid metabolite of VG \\
\hline M20.2 & $\begin{array}{l}2.8-3.5 \\
\quad \text { (broad) }\end{array}$ & $\mathrm{P}, \mathrm{U}$ & $\mathrm{U}, \mathrm{B}$ & $\begin{array}{l}\text { Pos: } 480.2335-45(-0.5 \text { to } 0.5) \\
\quad \text { Neg: } 478.2193-5(-0.2 \text { to } 0)\end{array}$ & $\mathrm{C}_{23} \mathrm{H}_{33} \mathrm{~N}_{3} \mathrm{O}_{8}$ & $O$-Glucuronide of VG \\
\hline Mono-hydroxyl VG-1 & $3.45-3.58$ & $\mathrm{P}, \mathrm{U}, \mathrm{F}$ & $\mathrm{U}, \mathrm{F}, \mathrm{B}$ & $\begin{array}{l}\text { Pos: } 320.1963-70 \text { (-0.6 to } 0.1) \\
\text { Neg: not detectable }\end{array}$ & $\mathrm{C}_{17} \mathrm{H}_{25} \mathrm{~N}_{3} \mathrm{O}_{3}$ & Mono-hydroxyl VG \\
\hline M18.6 & $3.55-3.68$ & $\mathrm{P}, \mathrm{U}, \mathrm{F}$ & $\mathrm{U}, \mathrm{F}, \mathrm{B}$ & $\begin{array}{l}\text { Pos: } 322.2117-26(-0.8 \text { to } 0.1) \\
\text { Neg: not detectable }\end{array}$ & $\mathrm{C}_{17} \mathrm{H}_{27} \mathrm{~N}_{3} \mathrm{O}_{3}$ & Amide metabolite of VG \\
\hline Mono-hydroxyl VG-2 & $3.83-3.98$ & $\mathrm{P}, \mathrm{U}, \mathrm{F}$ & $\mathrm{U}, \mathrm{F}, \mathrm{B}$ & $\begin{array}{l}\text { Pos: } 320.1961-8(-0.8 \text { to }-0.1) \\
\text { Neg: not detectable }\end{array}$ & $\mathrm{C}_{17} \mathrm{H}_{25} \mathrm{~N}_{3} \mathrm{O}_{3}$ & Mono-hydroxyl VG \\
\hline M407 & $3.86-3.96$ & $\mathrm{P}, \mathrm{U}, \mathrm{F}$ & $\mathrm{U}, \mathrm{F}, \mathrm{B}$ & $\begin{array}{l}\text { Pos: } 408.1942-54(-1.0 \text { to } 0.2) \\
\text { Neg: } 406.1796-811(-1.0 \text { to } 0.5)\end{array}$ & $\mathrm{C}_{20} \mathrm{H}_{29} \mathrm{~N}_{3} \mathrm{O}_{4} \mathrm{~S}$ & Cysteine conjugate of VG \\
\hline M464 & $4.01-4.09$ & $\mathrm{U}, \mathrm{F}$ & $\mathrm{U}, \mathrm{F}, \mathrm{B}$ & $\begin{array}{l}\text { Pos: } 465.2156-65(-1.0 \text { to }-0.1) \\
\text { Neg: } 463.2021(0)\end{array}$ & $\mathrm{C}_{22} \mathrm{H}_{32} \mathrm{~N}_{4} \mathrm{O}_{5} \mathrm{~S}$ & Cysteinylglycine conjugate of VG \\
\hline VG & $4.38-4.45$ & $\mathrm{P}, \mathrm{U}, \mathrm{F}$ & $\mathrm{U}, \mathrm{F}, \mathrm{B}$ & $\begin{array}{l}\text { Pos: } 304.2012-9(-0.8 \text { to }-0.1) \\
\text { Neg: not detectable }\end{array}$ & $\mathrm{C}_{17} \mathrm{H}_{25} \mathrm{~N}_{3} \mathrm{O}_{2}$ & VG \\
\hline
\end{tabular}

B, bile sample at $0-4,4-8$, and $8-24 \mathrm{~h} ; \mathrm{F}$, feces sample at $0-24 \mathrm{~h} ; \mathrm{Neg}$, negative ionization mode; $\mathrm{P}$, plasma sample 1,2 , and $4 \mathrm{~h}$ postdosing; Pos, positive ionization mode; $\mathrm{U}$, urine sample at $0-24 \mathrm{~h} ; \Delta$, the difference between measured accurate mass and calculated exact mass.

(Williams and Jaeschke, 2012; Uetrecht and Naisbitt, 2013). Covalent binding of chemicals to human liver microsomes at less than $50 \mathrm{pmol}$ $\mathrm{Eq} / \mathrm{mg}$ protein is considered to translate to a relatively low risk of toxicity (Evans et al., 2004). DILI risks have also been reported to be predictable using the extent of covalent binding to human hepatocytes or liver microsomes in combination with clinical daily doses (Nakayama et al., 2009; Sakatis et al., 2012).

GSH and its analogs are nucleophiles that trap soft electrophiles, such as epoxides or $\alpha, \beta$-unsaturated carbonyls, whereas cyanides or amines trap hard electrophiles such as aldehydes and iminium structures. The production of GSH conjugates of chemicals can be a marker of covalent binding of these chemicals to thiol residues of cysteine in protein (Gómez-Lechón et al., 2016). We recently found in vitro that VG is rapidly trapped by L-cysteine, rather than GSH, in a nonenzymatic manner to produce the thiazoline-containing cysteine adduct metabolite M407 (Mizuno et al., 2018). In this study, we sought to clarify whether the metabolite was actually formed in vivo. After fed male rats were treated with VG, in addition to M407, its glucuronide M583 and the cysteinylglycine adduct metabolite of VG M464 were observed in rats. M464, similar to M407, has a thiazoline moiety (Fig. 4). Further in vitro experiments demonstrated that M464 was generated from VG by coincubation with GSH in a nonenzymatic manner (Fig. 6), indicating that the thiazoline moiety of M464 was formed by the nucleophilic conjugation of a thiol residue of GSH to the nitrile prior to hydrolysis of the cysteine-glutamate bond of GSH and loss of the element of ammonia (Fig. 8). It was also suggested that another GSH adduct, namely M611,

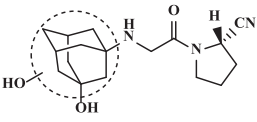

M14.9*<smiles>CC1C=CN(C(=O)CNC23CC4CC(CC(C4)C2)C3)CC1C</smiles>
M16.7*<smiles>O=C(O)CCC1CC2CCC(C1)O2</smiles>

M15.3*<smiles>CC1C2CC3CC(C2)C(NC(=O)CC(=O)N2CCCC2C(N)=O)C1C3</smiles>
M18.6*

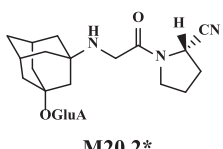

M20.2*
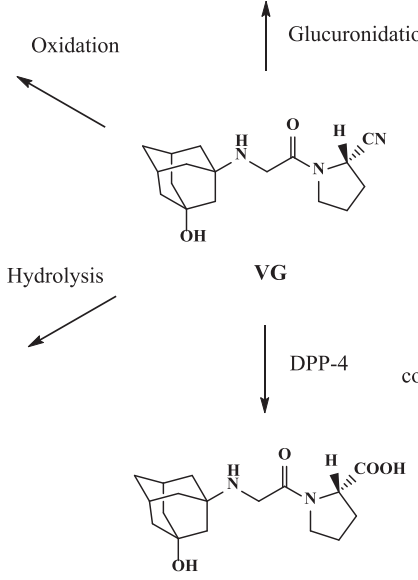

M20.7
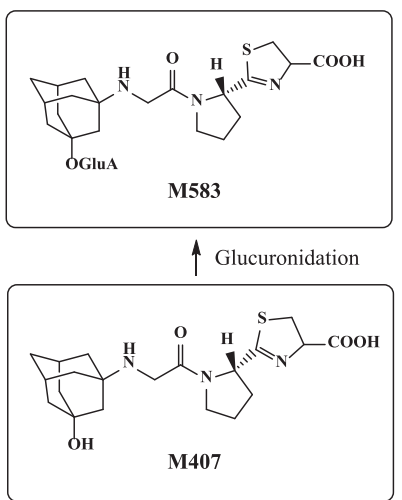

Fig. 4. Proposed metabolic pathways of VG in rats. *Derived from He et al. (2009b). 
A

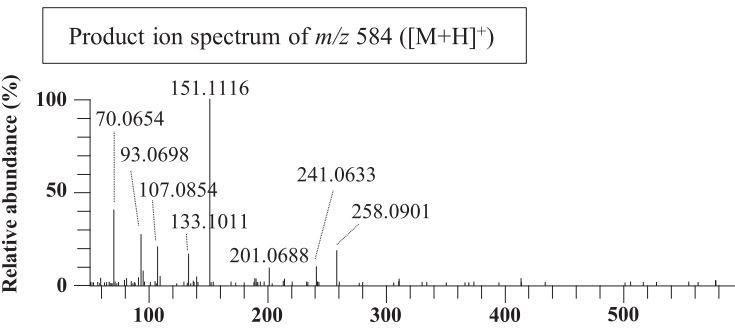

Product ion spectrum of $m / z 582\left([\mathrm{M}-\mathrm{H}]^{-}\right)$

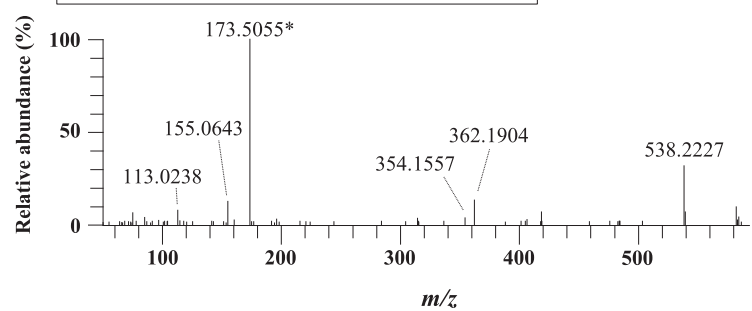

B

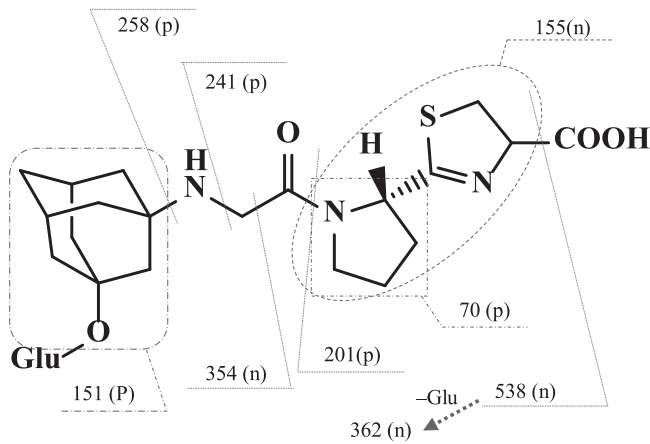

M583
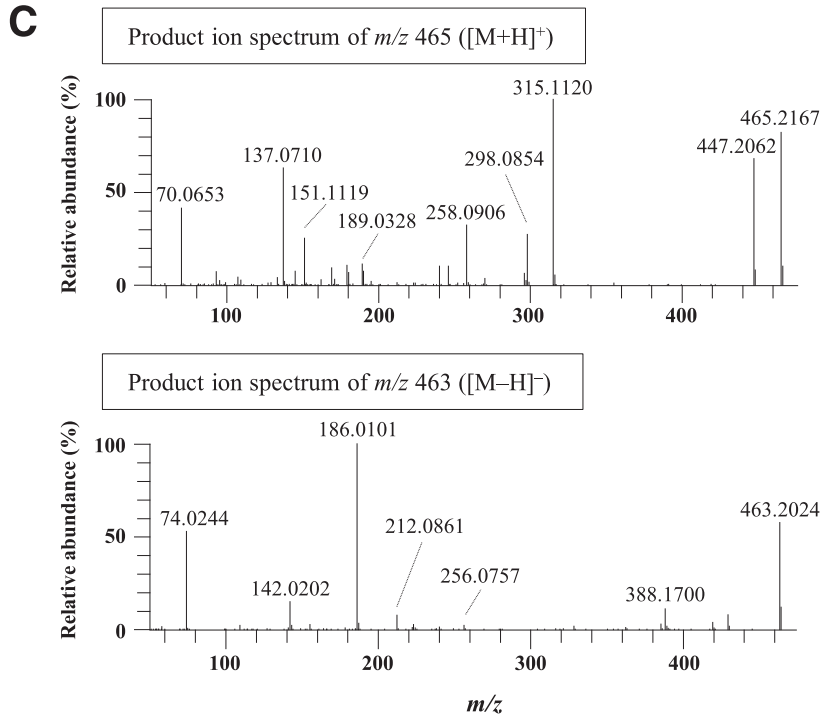

D

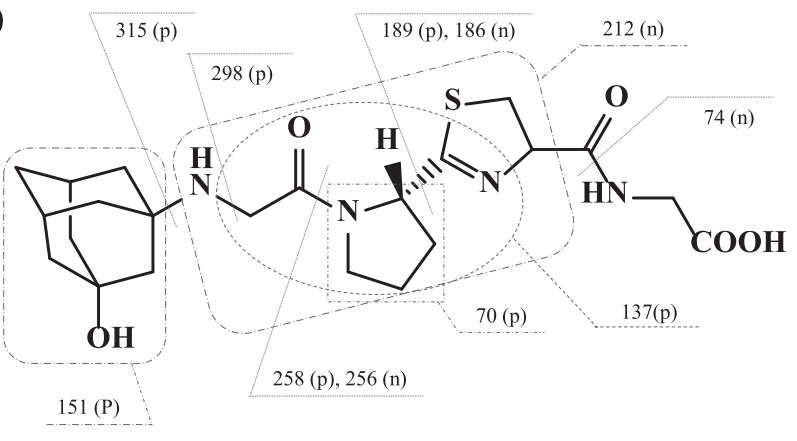

M464

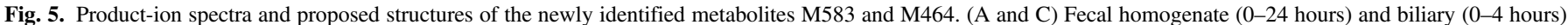

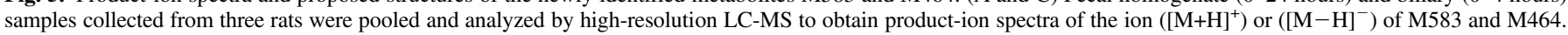
Proposed structures and fragmentations of M583 (B) and M464 (D). *Nonspecific interference peak.

was produced via GSH conjugation to the nitrile. The initial step of the molecular mechanism is therefore considered identical to that of M407 (Mizuno et al., 2018). Although VG was rapidly bound to L-cysteine (half-life, 20 minutes), VG was stable when incubated with GSH for at least 2 hours (Mizuno et al., 2018). In the present study, VG was incubated with GSH in the absence of metabolic enzymes for a longer period (24 hours), and M464 was detected (Fig. 6). This suggests that the in vivo production rate of M464 was lower than that of M407. In our study, three thiol adduct metabolites (M407, M583, and M464) were detected in rats, and these metabolites were produced via cysteine or GSH conjugation to VG nonenzymatically. It is possible that VG covalently binds to cysteine residues of proteins in humans in vivo, triggering adverse reactions.
Bile duct-cannulated rats are frequently used to estimate biliary elimination of compounds and to evaluate enterohepatic recirculation of drugs (Klauda et al., 1973; Bachir-Cherif et al., 2011). In the present study, we used bile duct-cannulated rats and found that biliary excretion of M407 was $0.03 \%$ of the dose after oral administration of VG (Fig. 3). Treatment of bile samples with $\beta$-glucuronidase significantly increased their M407 contents, and biliary excretion of its glucuronide, M583, was estimated to be $0.1 \%$ of the dose (Table 3). Because M583 itself was not detected in feces, we hypothesized that M583 secreted into bile was deconjugated to M407 in the intestine, followed by reabsorption or fecal excretion. Similar results were observed for VG and its glucuronide M20.2 (Fig. 3; Tables 2 and 3), indicating that M407 and VG circulate enterohepatically in the body.

TABLE 3

Biliary excretions of the metabolites M583 and M20.2 in bile duct-cannulated rats

Biliary samples obtained from VG-treated bile duct-cannulated rats were deconjugated using $\beta$-glucuronidase to calculate the biliary excretion of glucuronides. Data are shown as the mean \pm S.D. of three rats. Biliary excretion differed significantly $(\mathrm{P}<0.01)$ between incubations with and without $\beta$-glucuronidase (unpaired $t$ test).

\begin{tabular}{lccc}
\hline \multirow{2}{*}{ Analyte } & \multicolumn{4}{c}{ Biliary excretion (\% of dose) (analyzed compounds) } \\
\cline { 2 - 4 } & $\beta$-Glucuronidase(-) & $\beta$-Glucuronidase $(+)$ & $\Delta$ \\
\hline M407 & $0.0521 \pm 0.0084(\mathrm{M} 407)$ & $0.151 \pm 0.024(\mathrm{M} 407+\mathrm{M} 583)$ & $0.0984 \pm 0.0174(\mathrm{M} 583)$ \\
VG & $0.225 \pm 0.048(\mathrm{VG})$ & $33.1 \pm 5.7(\mathrm{VG}+\mathrm{M} 20.2)$ & $32.9 \pm 5.7(\mathrm{M} 20.2)$ \\
\hline
\end{tabular}

$\Delta$, the difference between incubation with and without $\beta$-glucuronidase. 
A

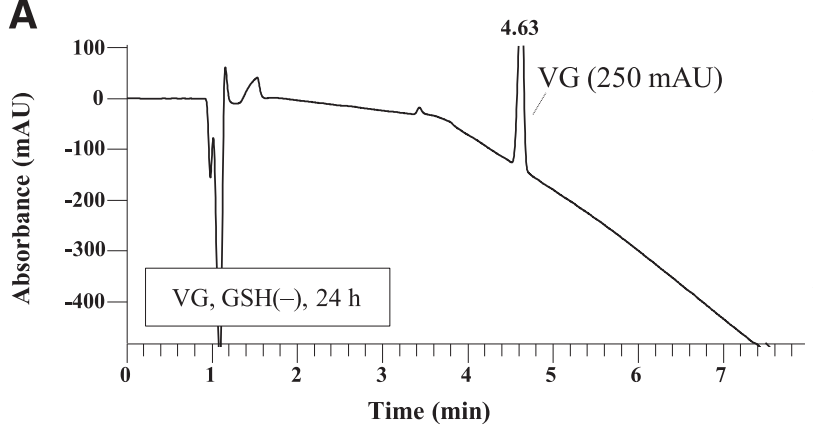

B
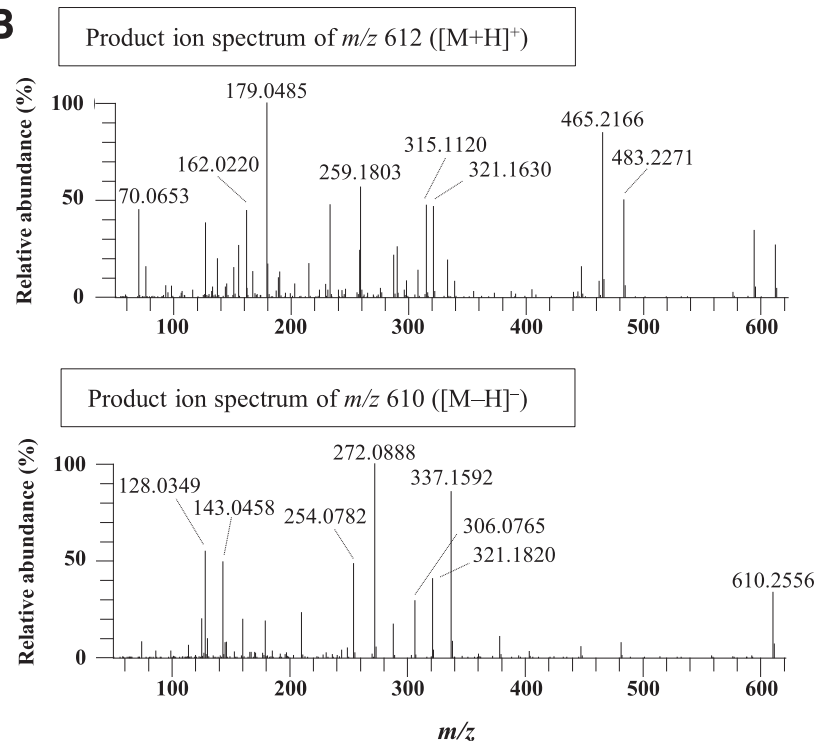

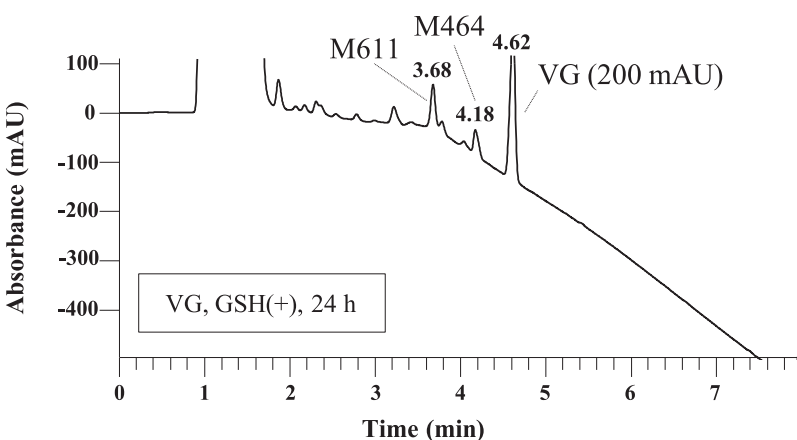

C

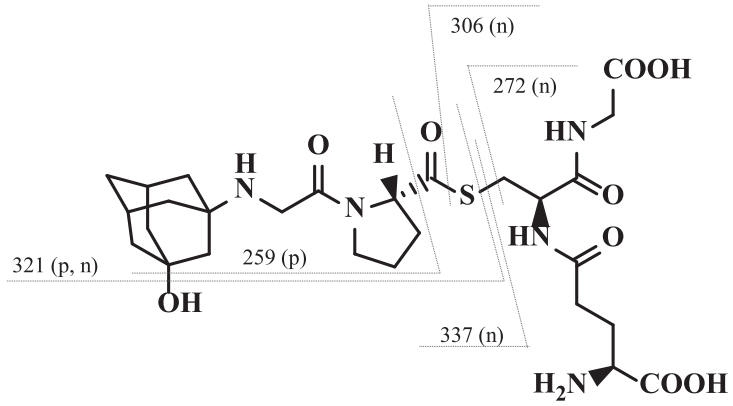

M611

Fig. 6. Formation of M464 and M611 by in vitro incubation of VG with GSH. (A) VG (100 $\mu \mathrm{M})$ was incubated with or without GSH (100 mM) at $37^{\circ} \mathrm{C}$ for 24 hours to

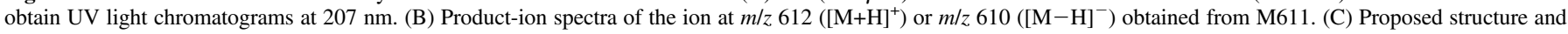
fragmentation of M611.

Some drugs bind covalently to the L-cysteine of proteins in humans. For example, anticancer drugs such as alkylating agents and $N$-acetyl- $p$-benzoquinoneimine (a reactive metabolite of acetaminophen) covalently bind to Cys34 in human serum albumin (Noort et al., 2002; Damsten et al., 2007). Human albumin is a major protein component of plasma, and contains 35 cysteine residues (Yamasaki et al., 2013). Although 34 residues form internal disulfides, the Cys34 in subdomain IA, which is located in a crevice on the surface of the protein, has been reported to participate in covalent binding with drugs and metabolites. Such drug-albumin complexes are observed in sera from patients or healthy volunteers receiving these drugs, suggesting that the complexes are useful markers for in vivo covalent binding in humans. In other cases, reactive metabolites of raloxifene covalently bind to Cys225 of CYP2C8 and Cys239 of CYP3A4 (Baer et al., 2007; VandenBrink et al., 2012). Because VG irreversibly binds to L-cysteine or GSH, it may also be capable of covalent binding to cysteine residues of proteins in humans and animals.
A

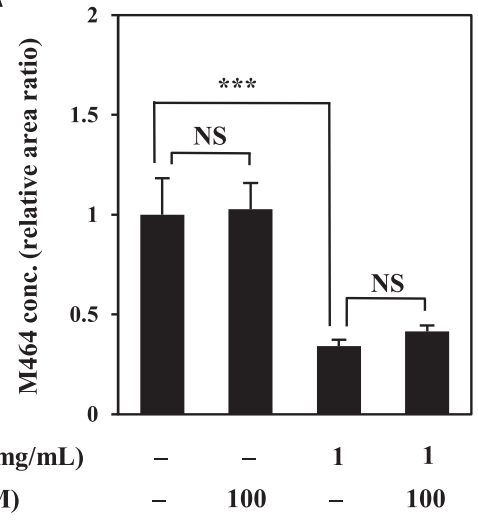

B

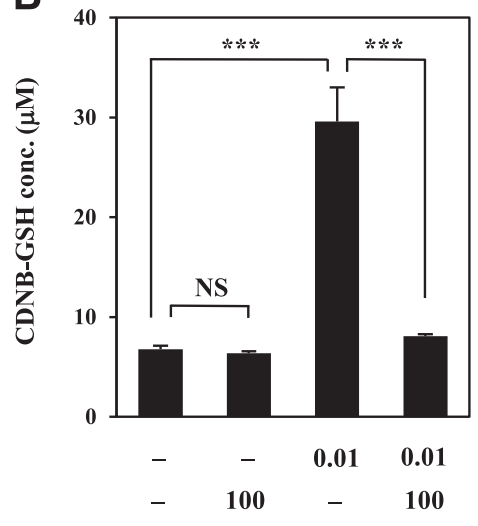

Fig. 7. Effects of human GST on M464 production from VG. (A) VG $(100 \mu \mathrm{M})$ was incubated with HLC $(1 \mathrm{mg} / \mathrm{ml})$, GSH $(5 \mathrm{mM})$, and EA $(100 \mu \mathrm{M})$ at $25^{\circ} \mathrm{C}$ for 30 minutes. (B) CDNB $(200 \mu \mathrm{M})$ was incubated with HLC $(0.01 \mathrm{mg} / \mathrm{ml})$, GSH $(5 \mathrm{mM})$, and EA $(100 \mu \mathrm{M})$ at $25^{\circ} \mathrm{C}$ for 5 minutes. Data are shown as the mean \pm S.D. of triplicate determinations. $* * * P<0.001$ (Tukey's test). NS, not significant.

$$
\begin{array}{lcccc}
\text { HLC }(\mathrm{mg} / \mathrm{mL}) & - & - & 1 & 1 \\
\operatorname{EA}(\mu \mathrm{M}) & - & 100 & - & 100
\end{array}
$$




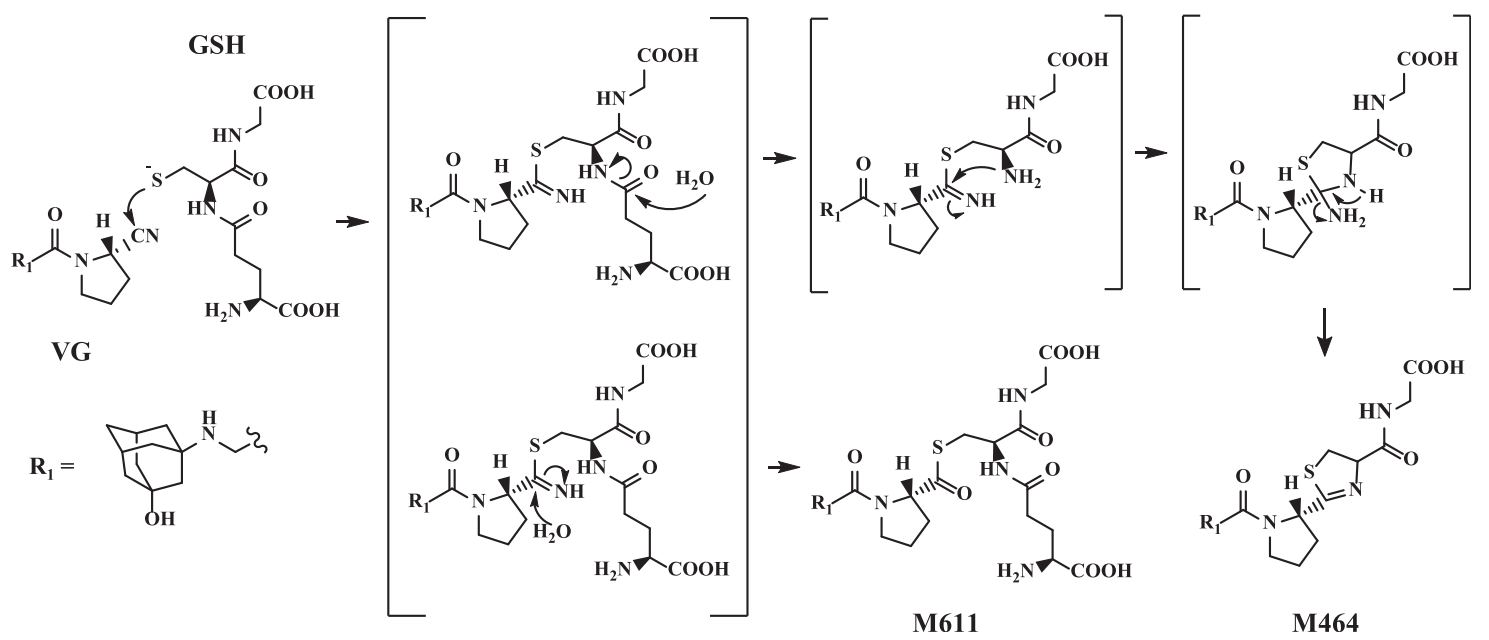

Fig. 8. Proposed mechanisms for irreversible covalent binding of GSH to VG.

Since the DLST was positive in a patient with VG-induced hepatotoxicity (Kurita et al., 2014), it was assumed that an immune response is associated with VG-induced liver injury. A patient with VG-induced fever was also DLST positive, and drug rechallenge resulted in worsening of the symptoms (Anno et al., 2019), supporting the hypothesis that VG can induce immune responses in susceptible patients. Because many causal mechanisms of immune-mediated DILI have been identified, multiple approaches would be required to understand the mechanisms of VG-induced hepatotoxicity. Previously, we demonstrated that M20.7, a major metabolite of VG (He et al., 2009a), is stable during coincubation with GSH or L-cysteine, suggesting that M20.7 is barely capable of covalent binding to proteins in humans (Mizuno et al., 2018). In contrast, Asakura et al. (2016) have reported that M20.7 and VG can release a complex of proinflammatory S100 calcium-binding protein A8 (S100A8) and S100A9 from human hepatoma HepG2 cells, thereby inducing a variety of inflammatory responses (Foell et al., 2007). Therefore, in addition to covalent binding of VG to proteins, the release of danger signals may be responsible for accreting immunologic reactions in patients susceptible to VG. Further clinical and preclinical approaches are warranted to elucidate the mechanisms of VG-induced liver injury and to clarify the relevant susceptibility factors.

Conclusion. After oral administration of VG, a thiazoline acidcontaining cysteine adduct metabolite M407 was detected in rat plasma, urine, feces, and bile. Its total excretion reached $2 \%$ of the dose. The glucuronide of M407, M583, was also detected in bile. A thiazolinecontaining GSH adduct metabolite, M464, was detected in urine, feces, and bile, and the formation of this metabolite was confirmed by in vitro incubation of VG with GSH. Our findings indicate that VG can bind covalently to cysteine residues of proteins in humans, implying the possibility of relevance as a cause of adverse drug reactions, as generally appreciated.

\section{Acknowledgments}

We thank Dean Meyer, ELS from Edanz Group (www.edanzediting.com/ac) for editing a draft of this manuscript.

\section{Authorship Contributions}

Participated in research design: Mizuno and Nakajima.

Conducted experiments, contributed new reagents or analytic tools, and performed data analysis: Mizuno.

Wrote or contributed to the writing of the manuscript: Mizuno, Takeuchi, Umehara, and Nakajima.

\section{References}

Anno T, Kaneto H, Kawasaki F, Shigemoto R, Aoyama Y, Kaku K, and Okimoto N (2019) Drug fever and acute inflammation from hypercytokinemia triggered by dipeptidyl peptidase-4 inhibitor vildagliptin. J Diabetes Investig 10:182-185.

Asakura M, Fujii H, Atsuda K, Itoh T, and Fujiwara R (2015) Dipeptidyl peptidase-4 greatly contributes to the hydrolysis of vildagliptin in human liver. Drug Metab Dispos 43:477-484.

Asakura M, Karaki F, Fujii H, Atsuda K, Itoh T, and Fujiwara R (2016) Vildagliptin and its metabolite M20.7 induce the expression of S100A8 and S100A9 in human hepatoma HepG2 and leukemia HL-60 cells. Sci Rep 6:35633.

Asakura M, Fukami T, Nakajima M, Fujii H, Atsuda K, Itoh T, and Fujiwara R (2017) Hepatic dipeptidyl peptidase-4 controls pharmacokinetics of vildagliptin in vivo. Drug Metab Dispos 45:237-245.

Bachir-Cherif D, Blum D, Braendli-Baiocco A, Chaput E, Pacheco GC, Flint N, Haiker M, Hoflack JC, Justies N, Neff R, et al. (2011) Characterization of post-surgical alterations in the bile ductcannulated rat. Xenobiotica 41:701-711.

Baer BR, Wienkers LC, and Rock DA (2007) Time-dependent inactivation of P450 3A4 by raloxifene: identification of Cys239 as the site of apoprotein alkylation. Chem Res Toxicol 20:954-964.

Burkey BF, Li X, Bolognese L, Balkan B, Mone M, Russell M, Hughes TE, and Wang PR (2005) Acute and chronic effects of the incretin enhancer vildagliptin in insulin-resistant rats. J Pharmacol Exp Ther 315:688-695.

Busch SJ, Hoffmann P, Sahota P, Johnson R, Kothny W, Meyer F, and Foley JE (2013) Studies in rodents with the dipeptidyl peptidase-4 inhibitor vildagliptin to evaluate possible drug-induced pancreatic histological changes that are predictive of pancreatitis and cancer development in man. Diabetes Obes Metab 15:72-76.

Dahal UP, Obach RS, and Gilbert AM (2013) Benchmarking in vitro covalent binding burden as a tool to assess potential toxicity caused by nonspecific covalent binding of covalent drugs. Chem Res Toxicol 26:1739-1745.

Damsten MC, Commandeur JN, Fidder A, Hulst AG, Touw D, Noort D, and Vermeulen NP (2007) Liquid chromatography/tandem mass spectrometry detection of covalent binding of acetaminophen to human serum albumin. Drug Metab Dispos 35:1408-1417.

Deacon CF and Lebovitz HE (2016) Comparative review of dipeptidyl peptidase-4 inhibitors and sulphonylureas. Diabetes Obes Metab 18:333-347.

Evans DC, Watt AP, Nicoll-Griffith DA, and Baillie TA (2004) Drug-protein adducts: an industry perspective on minimizing the potential for drug bioactivation in drug discovery and development. Chem Res Toxicol 17:3-16.

Foell D, Wittkowski H, Vogl T, and Roth J (2007) S100 proteins expressed in phagocytes: a novel group of damage-associated molecular pattern molecules. J Leukoc Biol 81:28-37.

Gómez-Lechón MJ, Tolosa L, and Donato MT (2016) Metabolic activation and drug-induced liver injury: in vitro approaches for the safety risk assessment of new drugs. J Appl Toxicol 36:752-768.

Habig WH, Pabst MJ, and Jakoby WB (1974) Glutathione S-transferases. The first enzymatic step in mercapturic acid formation. J Biol Chem 249:7130-7139.

Hayes JD, Flanagan JU, and Jowsey IR (2005) Glutathione transferases. Annu Rev Pharmacol Toxicol 45:51-88

He H, Tran P, Yin H, Smith H, Batard Y, Wang L, Einolf H, Gu H, Mangold JB, Fischer V, et al. (2009a) Absorption, metabolism, and excretion of [14C]vildagliptin, a novel dipeptidyl peptidase 4 inhibitor, in humans. Drug Metab Dispos 37:536-544.

He H, Tran P, Yin H, Smith H, Flood D, Kramp R, Filipeck R, Fischer V, and Howard D (2009b) Disposition of vildagliptin, a novel dipeptidyl peptidase 4 inhibitor, in rats and dogs. Drug Metab Dispos 37:545-554.

He YL (2012) Clinical pharmacokinetics and pharmacodynamics of vildagliptin. Clin Pharmacokinet 51:147-162.

Kaplowitz N (2005) Idiosyncratic drug hepatotoxicity. Nat Rev Drug Discov 4:489-499.

Klauda HC, McGovern RF, and Quackenbush FW (1973) Use of a bile duct T-cannula as a new technique for studying bile acid turnover in the rat. Lipids 8:459-465.

Kurita N, Ito T, Shimizu S, Hirata T, and Uchihara H (2014) Idiosyncratic liver injury induced by vildagliptin with successful switch to linagliptin in a hemodialyzed diabetic patient. Diabetes Care 37:e198-e199.

Lee WM (2003) Drug-induced hepatotoxicity. N Engl J Med 349:474-485.

Ligueros-Saylan M, Foley JE, Schweizer A, Couturier A, and Kothny W (2010) An assessment of adverse effects of vildagliptin versus comparators on the liver, the pancreas, the immune system, 
the skin and in patients with impaired renal function from a large pooled database of Phase II and III clinical trials. Diabetes Obes Metab 12:495-509.

Mathieu C, Kozlovski P, Paldánius PM, Foley JE, Modgill V, Evans M, and Serban C (2017) Clinical safety and tolerability of vildagliptin - insights from randomised trials, observational studies and post-marketing surveillance. Eur Endocrinol 13:68-72.

Mizuno K, Takeuchi K, Umehara K, and Nakajima M (2018) Identification of a novel metabolite of vildagliptin in humans: cysteine targets the nitrile moiety to form a thiazoline ring. Biochem Pharmacol 156:312-321.

Nakayama S, Atsumi R, Takakusa H, Kobayashi Y, Kurihara A, Nagai Y, Nakai D, and Okazaki O (2009) A zone classification system for risk assessment of idiosyncratic drug toxicity using daily dose and covalent binding. Drug Metab Dispos 37:1970-1977.

Noort D, Hulst AG, and Jansen R (2002) Covalent binding of nitrogen mustards to the cysteine-34 residue in human serum albumin. Arch Toxicol 76:83-88.

Sakatis MZ, Reese MJ, Harrell AW, Taylor MA, Baines IA, Chen L, Bloomer JC, Yang EY, Ellens HM, Ambroso JL, et al. (2012) Preclinical strategy to reduce clinical hepatotoxicity using in vitro bioactivation data for $>200$ compounds. Chem Res Toxicol 25 $2067-2082$

Stepan AF, Walker DP, Bauman J, Price DA, Baillie TA, Kalgutkar AS, and Aleo MD (2011) Structural alert/reactive metabolite concept as applied in medicinal chemistry to mitigate the risk of idiosyncratic drug toxicity: a perspective based on the critical examination of trends in the top 200 drugs marketed in the United States. Chem Res Toxicol 24:1345-1410.
Uetrecht J and Naisbitt DJ (2013) Idiosyncratic adverse drug reactions: current concepts. Pharmacol Rev 65:779-808.

VandenBrink BM, Davis JA, Pearson JT, Foti RS, Wienkers LC, and Rock DA (2012) Cytochrome p450 architecture and cysteine nucleophile placement impact raloxifene-mediated mechanismbased inactivation. Mol Pharmacol 82:835-842.

Villhauer EB, Brinkman JA, Naderi GB, Burkey BF, Dunning BE, Prasad K, Mangold BL, Russel ME, and Hughes TE (2003) 1-[[(3-hydroxy-1-adamantyl)amino]acetyl]-2-cyano-(S)-pyrrolidine: a potent, selective, and orally bioavailable dipeptidyl peptidase IV inhibitor with antihyperglycemic properties. J Med Chem 46:2774-2789.

Williams CD and Jaeschke H (2012) Role of innate and adaptive immunity during drug-induced liver injury. Toxicol Res 1:161-170.

Yamasaki K, Chuang VT, Maruyama T, and Otagiri M (2013) Albumin-drug interaction and it clinical implication. Biochim Biophys Acta 1830:5435-5443.

Address correspondence to: Katsuhiko Mizuno, Department of Drug Metabolism and Pharmacokinetics, Nonclinical Research Center, Tokushima Research Institute, Otsuka Pharmaceutical Co., Ltd., 463-10 Kagasuno, Kawauchi-cho, Tokushima 771-0192, Japan. E-mail: Mizuno.Katsuhiko@otsuka.jp 